\title{
MODELAGEM DOS PROCESSOS DE REMOÇÃO SULFATO E DIÓXIDO DE ENXOFRE PRESENTE NO PARTICULADO EM DIFERENTES LOCALIDADES DA REGIÃO METROPOLITANA DE SÃO PAULO
}

\author{
Fábio Luiz Teixeira Gonçalves ${ }^{1}$, Luiz Carlos Mantovani Junior ${ }^{1}$, \\ Adalgiza Fornaro ${ }^{1}$ e Jairo José Pedrotti ${ }^{2}$ \\ Recebido em 27 fevereiro, 2009 / Aceito em 5 janeiro, 2010 \\ Received on February 27, 2009 / Accepted on January 5, 2010
}

\begin{abstract}
This article deals with the scavenging processes modeling of the particulate sulfate and the gas sulfur dioxide, emphasizing the synoptic conditions at different sampling sites in order to verify the domination of the in-cloud or below-cloud scavenging processes in the Metropolitan Area of São Paulo (RMSP). Three sampling sites were chosen: GV (Granja Viana) at RMSP surroundings, IAG-USP and Mackenzie (RMSP center). Basing on synoptic conditions, it was chosen a group of events where the numerical modeling, a simple scavenging model, was used. These synoptic conditions were usually convective cloud storms, which are usual at RMSP. The results show that the in-cloud processes were dominant (80\%) for sulfate/sulfur dioxide scavenging processes, with below-cloud process indicating around $20 \%$ of the total. Clearly convective events, with total rainfall higher than $20 \mathrm{~mm}$, are better modeled than the stratiform events, with correlation coefficient of 0.92 . There is also a clear association with events presenting higher rainfall amount and the ratio between modeled and observed data set with correlation coefficient of 0.63 . Additionally, the suburb sampling site, GV, as expected due to the pollution source distance, presents in general smaller amount of rainwater sulfate (modeled and observed) than the center sampling site, Mackenzie, where the characterization event explains partially the rainfall concentration differences.
\end{abstract}

Keywords: scavenging processes, sulfate and sulfur dioxide, air pollution.

RESUMO. Este artigo versa sobre a modelagem dos processos de remoção do sulfato no material particulado sulfato e o gás dióxido de enxofre, comparando as condições sinópticas, em diferentes locais de coleta, com o intuito de se verificar a dominância dos processos dentro e abaixo da nuvem na Região Metropolitana de São Paulo (RMSP). Três locais de amostragens foram escolhidos: GV (Granja Viana) na periferia da RMSP, IAG-USP e no Mackenzie (RMSP centro). Baseando-se nas condições sinópticas, foram escolhidos grupos de eventos onde a modelagem numérica foi simulada, usando um modelo simples de remoção. Estas condições foram geralmente de nuvens convectivas, sendo as mais comuns na RMSP. Os resultados mostram que os processos dentro da (intra-)nuvem foram dominantes (80\%) para a remoção de sulfato/dióxido de enxofre. Os processos de remoção abaixo da nuvem indicaram cerca de $20 \%$ deste total. Eventos claramente convectivos, com precipitação total maior que 20 mm, são mais eficientemente modelados que os eventos mais estratiformes, com um coeficiente de correlação de 0,92. Há também uma clara associação entre eventos de maior precipitação e a razão entre modelado e observado, com coeficiente de correlação de 0,63. Adicionalmente, como seria de esperar a respeito da distância às fontes de poluição, o local de amostragem GV apresentou de modo geral menores concentrações de sulfato na água de chuva, tanto modelado como observado, do que a região central, no Mackenzie, onde a caracterização dos eventos explica parcialmente as diferenças entre as concentrações em água de chuva.

Palavras-chave: processos de remoção, sulfato e dióxido de enxofre, poluição atmosférica.

\footnotetext{
${ }^{1}$ Departamento de Ciências Atmosféricas - IAG-USP, Rua do Matão, 1226, Cidade Universitária, 05508-090 São Paulo, SP, Brasil. Tel.: +55(11) 3091-4704; Fax:+55(11) 3091-4714-E-mails: fgoncalv@model.iag.usp.br; mantovani@model.iag.usp.br; fornaro@model.iag.usp.br

2 Universidade Presbiteriana Mackenzie, Departamento de Química, Rua da Consolação, 896, 01302-907, São Paulo, SP, Brasil. Tel.: +55(11) 2114-8145; Fax: +55(11) 2114-8147 - E-mail: jpedrotti@mackenzie.com.br
} 


\section{INTRODUÇÃo}

A poluição atmosférica e seus impactos nos ecossistemas são considerados um dos problemas tópicos no mundo atual. A poluição pode tanto ser de origem antrópica, como também proveniente de emissões naturais, devido à decomposição efetuada pela microbiota, e de erupções vulcânicas, entre outras fontes. Há vários processos atmosféricos que promovem a remoção da poluição atmosférica, dos quais a chuva tem papel relevante. A ocorrência de chuva contendo compostos poluentes e seus efeitos sobre os ecossistemas tem reconhecida importância desde a Antigüidade Clássica. Na Conferência Acid Reign '2005?, realizada na República Tcheca, houve diversos trabalhos na Europa Oriental demonstrando que houve significativa melhora da qualidade do ar na região, em particular do $\mathrm{SO}_{2}$, e conseqüente diminuição da acidez da chuva, e da concentração de sulfato (Christopher et al., 2007; Nogushi et al., 2007; Terauda \& Nikodemus, 2007).

Os hidrometeoros (aqui englobando gotas de chuva, nevoeiro, neve, granizo e graupe/) são os principais agentes dos processos de remoção da poluição da atmosfera, transferindo-a do reservatório atmosfera para a hidrosfera e litosfera, com seus óbvios impactos sobre a biosfera.

Os poluentes atmosféricos podem ser encontrados nas três fases, isto é, nos três estados da matéria: sólido, líquido e gasoso. A complexidade dos ciclos dos poluentes é resultado de interações entre estas diferentes fases, somados aos processos físicos (meteorológicos). Os processos de deposição atuam como uma conexão entre as fontes e sumidouros, controlando a concentração e 0 tempo de residência tanto para gases como para 0 aerossol. $0 \mathrm{SO}_{2}$ e 0 correspondente particulado sulfato estão entre os principais gases e aerossóis dentro do contexto da poluição do ar. Os mecanismos de remoção podem ser agrupados em deposição seca e úmida. A deposição úmida está sempre associada às gotas de chuva ou de nuvem, englobando os processos de adsorção de gotículas e/ou gotas, ainda dentro da nuvem (denominada em inglês por "in-cloud scavenging") ou ainda dentro de nevoeiros, seguidas pela remoção pela precipitação ("below-cloud" - abaixo da nuvem).

Sob perspectiva histórica, Chamberlain (1953) apresenta um primeiro tratamento para 0 estudo dos processos abaixo da nuvem, mostrando que a intensidade da precipitação e a localização das nuvens determinam o potencial de atuação desses processos. Hill \& Adamowics (1977) foram os primeiros a elaborar um modelo de remoção por gotas de chuva na região de Nova York. Neste último trabalho, modelou-se o processo abaixo da nuvem dos gases $\mathrm{SO}_{2}, \mathrm{NH}_{3}$ e CO $\mathrm{CO}_{2}$, em uma atmosfera poluída ea formação de sulfato dentro das gotas de chuva. Diversos autores, entre eles Pruppacher \& Klett (1997) mostraram que os seguin- tes íons são tipicamente encontrados em águas de nuvens e de chuva: $\mathrm{NH}_{4}^{+}, \mathrm{K}^{+}, \mathrm{Ca}^{2+}, \mathrm{Mg}^{2+}, \mathrm{H}^{+}, \mathrm{SO}_{4}^{2-}, \mathrm{Cl}^{-}, \mathrm{NO}_{3}^{-}, \mathrm{SO}_{3}^{2-}$, $\mathrm{HSO}_{3}^{-}, \mathrm{HCO}_{3}^{-}$e $\mathrm{CO}_{3}^{2-}$. Estudos da remoção do gás $\mathrm{SO}_{2}$ e do material particulado (MP), contendo sulfato em diferentes massas de ar, mostraram que as razões entre gás/MP são importantes para a concentração iônica na água de chuva (Okita et al., 1996).

Quanto à modelagem numérica dos processos de remoção há diversos trabalhos desde a década de 80, por exemplo, Herbert \& Beheng (1986), através do modelo denominado doravante de BV2 (Gonçalves et al., 2002, 2003, 2007a), simularam efeitos das interações de remoção de material particulado e gases por gotas de chuva. Devido ao fato de que a quantidade de material particulado e gases, capturados por hidrometeoros, dependerem de complexas interações entre ambos, foram utilizadas parametrizações desses processos dentro de um modelo numérico de processos de remoção abaixo da nuvem. Este modelo foi baseado em Pruppacher \& Klett (1997).

Outros trabalhos importantes envolvendo estudos de processos de remoção de poluentes com respeito à contribuição dentro e/ou abaixo da nuvem foram publicados nos últimos anos (Asman, 1995; Andronache, 2002; Mircea et al., 2004; Calderón et al., 2007), com resultados bastante diversos entre ambas as contribuições.

A região metropolitana de São Paulo (RMSP), além das diversas conseqüências nefastas decorrentes do fato de ser um grande centro urbano, tais como engarrafamentos, barulho entre outros, possui graves problemas ambientais, sendo a poluição atmosférica um dos seus mais pertinentes. Dentro do âmbito deste problema, o papel dos processos de remoção desta poluição por gotas de chuva e nuvem é largamente evidente, removendo os poluentes da atmosfera para a hidrosfera. Resultados de modelagem numérica associada à coleta de águas de chuva indicaram que a remoção dos poluentes atmosféricos, durante a estação chuvosa, é dominada pelo below-cloud para a cidade de S. Paulo e da Serra do Mar adjacente (Gonçalves et al., 2002, 2007a).

Estudos da composição química de águas de chuva da cidade de São Paulo iniciaram-se já na década de oitenta e noventa (Moreira-Nordemann et al., 1983; Forti et al., 1990). Apesar de terem sido realizados por diferentes grupos, os quais adotaram diferentes metodologias, foi possível observar-se a diminuição da acidez da precipitação ao longo destes últimos anos. Resultados de valores médios de pH ao redor de 4,5 (média ponderada pelo volume, MPV) foram observados nos estudos referentes ao período até 1995 (Fornaro et al., 2003). É importante lembrar que valores médios de pH são obtidos das médias de concentração de ions $\mathrm{H}^{+}\left(\mathrm{pH}=-\log \left[\mathrm{H}^{+}\right]\right)$, enquanto que resultados para amostras avaliadas no período entre 2002 e 2004 apresentaram va- 
lores de pH de 5,2 (MPV), ou seja, 5 vezes menos ácidas do que nos anos 80 e início da década de 1990, segundo Santos et al. (2007). Esta variabilidade da acidez foi associada, predominantemente, a diminuição das concentrações atmosféricas de $\mathrm{SO}_{2}$ (fase gasosa) e, conseqüentemente, de $\mathrm{SO}_{4}^{2-}$ nas águas de chuva (Fornaro \& Gutz, 2006). Outra característica importante da composição química de águas de chuva de São Paulo tem sido a presença marcante de íons amônio $\left(\mathrm{NH}_{4}^{+}\right)$, cuja presença na atmosfera está associada ao gás amônia $\left(\mathrm{NH}_{3}\right)$, cujo principal processo de remoção é a deposição úmida devido a sua alta solubilidade $\left(\mathrm{KH}=5,7 \times 10^{-4} \mathrm{~mol} \mathrm{~L}^{-1} \mathrm{~Pa}^{-1}\right)$. Outras espécies encontradas nas águas de chuva de São Paulo têm sido os íons nitrato $\left(\mathrm{NO}_{3}^{-}\right)$, sódio $\left(\mathrm{Na}^{+}\right)$cloreto $\left(\mathrm{Cl}^{-}\right)$, cálcio $\left(\mathrm{Ca}^{2+}\right)$, potássio $\left(\mathrm{K}^{+}\right)$, magnésio $\left(\mathrm{Mg}^{2+}\right)$, e também os ânions dos ácidos carboxílicos (acético, fórmico e oxálico). Sendo que estas últimas espécies têm apresentado importante contribuição potencial para a acidez destas amostras (Fornaro \& Gutz, 2006; Santos et al., 2007).

Como conseqüência da poluição, há diversos trabalhos na RMSP evidenciando seu impacto na saúde humana tanto em doenças cardio-vasculares (Sharovsky, 2001 e Gonçalves et al., 2007b) como respiratórias (Saldiva et al., 1992; Braga et al., 2000). Todas associadas à presença na atmosfera de sulfato $e$ dióxido de enxofre.

Conseqüentemente, a modelagem numérica dos processos deposição úmida, ou remoção de poluentes por hidrometeoros, é um tema de grande importância, principalmente no que concerne 0 sulfato. Este tema vem sendo abordado nos supracitados trabalhos de Gonçalves et al. (2002, 2003 e 2007a) e Nakaema (2002), para a RMSP e Amazônia (Silva et al., 2009) com contínuo aprimoramento da modelagem, incluindo-se efeitos intra-nuvens, maior detalhamento do espectro de gotas e particulados e, coletas em mais de um local para comparações intra e abaixo da nuvem, entre outras. Deve-se salientar que no trabalho de Gonçalves et al., 2007a, foi estudado somente um evento em duas localidades, por esta razão, este trabalho foca em um aumento considerável de eventos e localidades.

Portanto, este trabalho visa dar continuidade a este aprimoramento, verificando os processos de remoção, no caso do particulado sulfato e do gás $\mathrm{SO}_{2}$, em diversos eventos distintos em duas diferentes localidades da RMSP de modo a verificar a dominância de processos dentro e abaixo da nuvem e a eficiência da modelagem numérica e a diferença entre as localidades.

\section{METODOLOGIA}

A metodologia deste trabalho está dividida em três sessões: 2.1 - Levantamento de dados e caracterização sinótica dos dias de interesse; 2.2 - Modelagem dos processos de remoção com a obtenção e análise de dados através da modelagem de remoção de poluição, 2.3 - Metodologia da análise química

Os locais de amostragem foram: região central, no Colégio Mackenzie, na Consolação $\left(23^{\circ} 32,35^{\prime}\right.$ S e $\left.46^{\circ} 39,08^{\prime} \mathrm{W}\right)$; Cidade Universitária $\left(23^{\circ} 34^{\prime} \mathrm{S}\right.$ e $\left.46^{\circ} 44^{\prime} \mathrm{W}\right)$ e Granja Viana $\left(23^{\circ} 33,96^{\prime} \mathrm{S}\right.$, $46^{\circ} 52,45^{\prime} \mathrm{W}$ ), esta a uma altitude de $780 \mathrm{~m}$ (ver Fig. 1). Este último ponto de amostragem não possui fontes locais, estando fora da mancha urbana como se pode constar na Figura 1, enquanto que os outros dois pontos estão próximos a áreas de intenso trafego veicular. A Cidade Universitária é caracterizada por grande campus, com uma considerável área verde, mas rodeada de grandes vias de tráfego. 0 Colégio Mackenzie, por sua vez, se encontra próximo a vias de intenso tráfego, como a Av. Consolação, em uma região bastante central na RMSP. Os dados coletados na Granja Viana foram especificamente para este trabalho, os demais estão apresentados em trabalhos como Leal et al. (2004) e Santos et al. (2007), onde todos os detalhes metodológicos são fornecidos.

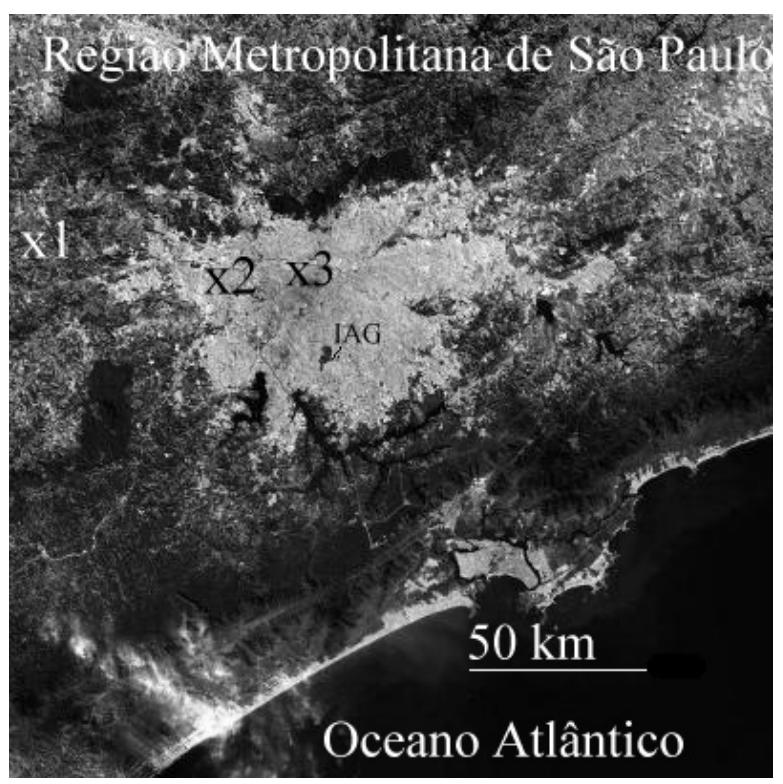

Figura 1 - Mapa da região da RMSP estudada com os locais de coleta: 1 Granja Viana, 2 - IAG-USP e 3 - Mackenzie e o IAG-Água Funda.

Os dados meteorológicos referentes à precipitação pluviométrica foram coletados na Estação Meteorológica, localizada na Água Funda, zona Sul de São Paulo. Em termos geográficos, situa-se na latitude de $23^{\circ} 39^{\prime} S$ e a longitude $46^{\circ} 37^{\prime}$ W e está no mesmo lugar desde 0 início de suas atividades em 22 de novembro de 1932. 


\section{Levantamento de dados e caracterização sinótica dos dias de interesse}

Primeiramente foram obtidos dados de observação de condições do tempo pela aeronáutica (mensagens METAR - cartas meteorológicas da Aeronáutica) fornecidas pelo aeroporto de Congonhas em São Paulo para os dias em que ocorreram os eventos de chuva a serem estudados. As mensagens METAR forneceram também quantitativamente a nebulosidade a cada hora do dia e, quando ocorreu, chuva, tempestades, nuvens convectivas em desenvolvimento (grandes formações), névoa úmida, granizo dentre outros fenômenos atmosféricos.

Os dados de umidade relativa utilizados para a simulação foram obtidos através da Estação Ibirapuera da CETESB e calculada como a média aritmética entre os valores obtidos e 100\% (umidade relativa na base da nuvem). Foram obtidas imagens de satélite (série temporal) nos canais infravermelho e visível do satélite GOES12 para os dias dos eventos, fornecidos pelo laboratório MASTER. As imagens auxiliaram na identificação de frentes frias as quais forneceram informações sobre a circulação geral atuante, para os dias de interesse.

As concentrações iniciais dos poluentes no ar, no caso 0 dióxido de enxofre, foram fornecidas pelas estações da CETESB mais próximas aos locais de onde foram colhidas as amostras de água de chuva (Estação Parque D. Pedro, próxima ao Mackenzie, Estação Osasco, a mais próxima do bairro Granja Viana e estação Ibirapuera para o IAG-USP, todas com menos de $10 \mathrm{~km}$ de distância entre elas).

Para 0 ânion sulfato a concentração inicial no ar foi estimada como sendo 20\% do valor medido do material particulado (Ynoue \& Andrade, 2004) do MP10, para as mesmas estações.

\section{Modelagem dos processos de remoção Cálculo do coeficiente de remoção do $\mathrm{SO}_{4}^{2-}$ e do $\mathrm{SO}_{2}$}

Para o cálculo do coeficiente de remoção do material particulado, tanto abaixo como dentro da nuvem, considerou-se que uma gota de água líquida em sua trajetória descendente através do ar, termina por coletar parte das partículas que encontra no seu caminho, logo, a concentração do poluente na atmosfera dentro (ou abaixo) da nuvem decresce com o tempo devido à remoção pelas gotículas e gotas. 0 coeficiente de remoção é introduzido no modelo de remoção, descrito a seguir.

0 número de partículas de diâmetro $D_{p}$ coletadas no tempo $d t$ é o produto do volume de colisão pelo número de partículas existente $\left(n\left(D_{g}\right) d D_{g}\right)$, no entanto, devido ao fluxo aerodinâmico, algumas partículas, ao entrar no mesmo, não colidem com a gota precipitante. A colisão depende de vários fatores, como, tamanho das gotas e das partículas, velocidade terminal de queda de ambas e de suas massas. Segundo Seinfeld \& Pandis (1998), considerando que os diâmetros das partículas e suas respectivas velocidades terminais são bem menores que as das gotas de chuva, os coeficientes de remoção do particulado sulfato, neste caso, abaixo e dentro da nuvem, $\Lambda\left(D_{p}\right) \mathrm{em} \mathrm{s}^{-1} \mathrm{e}$ $\beta\left(D_{p}\right)$, em s ${ }^{-1}$ são dados pelas equações:

$$
\begin{aligned}
\Lambda\left(D_{p}\right) & =\int \frac{\pi}{4} D_{g}^{2} V_{t} E\left(D_{p}, D_{g}\right) n\left(D_{g}\right) d D_{g} \\
\beta\left(D_{p}\right) & =\int \frac{\pi}{4} D_{p}^{2} v_{t} K\left(D_{p}, d_{g}\right) n\left(d_{g}\right) d d_{g}
\end{aligned}
$$

onde $D_{p}=$ diâmetro da partícula; $D_{g}=$ diâmetro da gota; $d_{g}=$ diâmetro da gotícula; $V_{t}=$ velocidade terminal da gota; $v_{t}=$ velocidade terminal da gotícula; $E\left(D_{p}, D_{g}\right)=$ eficiência de coleta das gotas; $K\left(D_{p}, d_{g}\right)=$ eficiência de coleta das gotículas; $n\left(D_{g}\right) d D_{g}=$ função de distribuiç̧ão de tamanho de gotas coletadas por intervalo de tamanho e; $n\left(d_{g}\right) d d_{g}=$ função de distribuição de tamanho de gotículas coletadas por intervalo de tamanho, conforme Seinfeld \& Pandis (1998).

Para 0 coeficiente de remoção dos gases a equação, tanto abaixo como dentro, é dado por:

$$
\Lambda\left(D_{g}\right)=\pi D_{g}^{2} k_{c} N\left(D_{g}\right)
$$

onde $k_{c}$ é 0 coeficiente de transferência de massa (pode ter as unidades em CGS: $\mathrm{cm} \cdot \mathrm{s}^{-1}$ ). Mais detalhes da modelagem dos processos de remoção e a descrição do BV.2 estão em Gonçalves et al. (2002, 2003 e 2007a).

A taxa de precipitação, mm. $\mathrm{h}^{-1}$, foi calculada através do valor da precipitação total (em mm, observada no IAG-Água Funda) dividido pelo tempo de duração do evento (igualmente observada no IAG-Água Funda, ver Tab. 1). Nesta tabela também é mostrada a precipitação acumulada no IAG (Água Funda).

0 modelo BV.2 simula os processos físicos responsáveis pela remoção de poluentes que estão abaixo da nuvem. A saída do modelo fornece 0 valor esperado de concentração dos íons do poluente, no caso 0 sulfato, na água de chuva em $\mu \mathrm{g} \cdot \mathrm{m}^{-3}$, podendo ser convertido em $\mu$ mol.L ${ }^{-1}$, para comparação ao observado. Para a modelagem no interior da nuvem, o BV.2 foi adaptado para tal fim, simulando os processos físicos que ocorrem na mesma, tais como nucleação. Este modelo utiliza basicamente as mesmas entradas do modelo anterior com a diferença de que a variável HZT (altura da base da nuvem em metros) agora é a variável do tamanho vertical da nuvem (em metros) e as demais variáveis referem-se à base da nuvem. A saída deste modelo 
também é dada em $\mu \mathrm{g} \cdot \mathrm{m}^{-3}$ e assim também é necessária a conversão. A saída é a mesma do caso de abaixo da nuvem.

Tabela 1 - Procedência dos dados utilizados neste estudo.

\begin{tabular}{|l|l|}
\hline Dado & Origem \\
\hline $\mathrm{SO}_{4}^{(-2)}$ na água $\left(\mathrm{mg}^{\left.-\mathrm{L}^{-1}\right)}\right.$ & LAPAt IAG-USP \\
\hline $\mathrm{SO}_{2} \mathrm{no}$ ar $\left(\mu \mathrm{g} / \mathrm{m}^{3}\right)$ & Estação de medição da CETESB \\
\hline $\mathrm{MP10}\left(\mu \mathrm{g} / \mathrm{m}^{3}\right)$ & Estação de medição da CETESB \\
\hline UR(\%) & Estação de medição da CETESB \\
\hline Temperatura $(\mathrm{K})$ & METAR de Congonhas \\
\hline Pressão $(\mathrm{hPa})$ & METAR de Congonhas \\
\hline Taxa de precipitação $(\mathrm{mm} / \mathrm{h})$ & $\begin{array}{l}\text { Precipitação em mm de chuva e } \\
\text { dividido pelo tempo de duração } \\
\text { da precipitação (obtidos no IAG- } \\
\text { Água Funda) }\end{array}$ \\
\hline
\end{tabular}

Uma consideração que foi feita, nas simulações dos processos intra-nuvem, é de que os tempos dos eventos são maiores do que os que foram utilizados para o modelo abaixo da nuvem. Isto se deveu ao fato de que os processos intra-nuvem agem antes de a chuva precipitar (formação e desenvolvimento da nuvem de chuva).

\section{Dados de entrada no BV.2}

A procedência dos dados apresentados, utilizados tanto para a modelagem intra-nuvem quanto para a modelagem abaixo da nuvem, estão na Tabela 1. As datas envolvidas neste trabalho estão apresentadas na Tabela 2 com respectivos locais, duração do evento e concentrações no ar e na água observadas.

Para o modelo de remoção intra-nuvem foi acrescentado 0 tempo de 180 minutos em cada duração, de acordo com 0 trabalho de Nakaema (2002). Foi considerado também um tamanho padrão vertical de nuvem de $8000 \mathrm{~m}$, com a base situada a $2000 \mathrm{~m}$. Estes resultados foram utilizados preliminarmente, baseados na média das simulações de diversos eventos meteorológicos na dissertação de mestrado de Nakaema (2002) e os artigos Gonçalves et al. (2002) e Gonçalves et al. (2007a). Estas simulações foram efetuadas com o modelo de mesoscala RAMS e serviram para os cálculos dos mesmos processos de remoção.

\section{Análise química de água de chuva pelo Laboratório de Processos Atmosféricos (LAPAt)}

No presente estudo para as análises do $\mathrm{SO}_{4}^{2-}$, na fase aquosa do material particulado foi utilizado o Cromatógrafo Metrohm mo- delo 761 com detecção condutométrica. Condições analíticas para determinação dos ânions foram: coluna aniônica Metrosep A-Supp5 $(250 \mathrm{~mm} \times 4 \mathrm{~mm})$, solução eluente de $\mathrm{Na}_{2} \mathrm{CO}_{3} 4,0$ $\mathrm{mmol} \mathrm{L}^{-1} / \mathrm{NaHCO}_{3} 1,0 \mathrm{mmol} \mathrm{L}^{-1}$; vazão de $0,7 \mathrm{ml} \mathrm{min}^{-1}$; coluna supressora Metrohm e regenerante solução de $\mathrm{H}_{2} \mathrm{SO}_{4}$

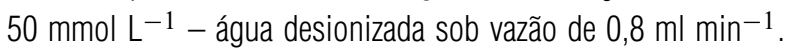
A qualidade dos dados está apresentada em trabalhos dos supracitados Leal et al. (2004) e Santos et al. (2007).

\section{RESULTADOS}

Os resultados estão abaixo relacionados com respeito à modelagem dos processos abaixo e dentro da nuvem, para todos os eventos e localidades, na sessão 3.1 e com respeito à comparação entre as localidades, na sessão 3.2.

\section{Resultados da modelagem de processos abaixo da nuvem e intra-nuvem}

Na Tabela 3 são mostradas as concentrações de sulfato observadas e modeladas, tanto dentro como abaixo da nuvem do sulfato, encontrado em água de chuva, e sua razão entre observado e modelado. Primeiramente, os resultados obtidos através da modelagem de somente processos abaixo da nuvem mostram-se abaixo dos observados, indicando que há outros processos envolvidos. Em média, os resultados modelados abaixo da nuvem foram somente $6,7 \pm 5,2 \%$ do total observado, com um máximo de 16\% ocorrido no dia 20/jan/05, na GV. Conseqüentemente, nesta tabela mostra-se claramente que os resultados dos valores das concentrações obtidas através da modelagem de processos intra-nuvens. Estas são significativamente e sistematicamente maiores que os obtidos com a modelagem abaixo da nuvem (por um fator de aproximadamente 4,6), como fica evidente na média de $31,0 \pm 25,4 \%$ do observado e um máximo de $76 \%$ no dia 22/fev/04, também na localidade GV (Tab. 2). Portanto, os processos no interior da nuvem possivelmente são responsáveis pela maior parte da remoção de poluição nestes eventos.

Enfatizando esta dominância, quando ambos os processos são inclusos, em quatro eventos, onde, em pelo menos uma das estações, o modelado chega a passar de $40 \%$ do valor observado (Tab. 2). Todos os quatro eventos são claramente convectivos, com precipitação total maior do que $20 \mathrm{~mm}$. Observa-se que a correlação entre as precipitações acumuladas e a razão entre observado e modelado é de 0,63 ( $P<0,05$, Fig. 2), para todos os eventos, evidenciando que há uma relação entre eventos mais claramente convectivos (ver discussão abaixo) e a melhor modelagem numérica. Portanto, observar-se na Figura 2, que em 
Tabela 2 - Dados utilizados para as simulações nos modelos de remoção de poluição. GV significa Granja Viana e CM, Consolação/Mackenzie. IAG-USP está localizado no Campus da USP. Observar que os dados apresentados para o sulfato na água de chuva estão em $\mu \mathrm{mol} . \mathrm{L}^{-1}$ e que a precipitação acumulada foi registrada no IAG-Água Funda.

\begin{tabular}{|c|c|c|c|c|c|c|c|}
\hline Dia & $\begin{array}{l}\text { Duração } \\
\text { (min) }\end{array}$ & Local & $\begin{array}{c}\mathrm{SO}_{4}^{2-} \\
\left(\mu \mathrm{mol} \cdot \mathrm{L}^{-1}\right)\end{array}$ & $\begin{array}{c}\mathrm{SO}_{2} \\
\left(\mu \mathrm{g} \cdot \mathrm{m}^{-3}\right)\end{array}$ & $\begin{array}{c}20 \% \text { do } \\
\text { MP10 } \\
\left(\mu \mathrm{g}^{-3} \mathrm{~m}^{-3}\right)\end{array}$ & $\begin{array}{c}\text { Volume } \\
\text { de chuva } \\
(\mathrm{ml})\end{array}$ & $\begin{array}{c}\text { Precipitação } \\
(\mathrm{mm})\end{array}$ \\
\hline \multirow{2}{*}{ 12/fev/04 } & 30 & GV & 28,40 & 14,50 & 3,98 & 30 & 1,5 \\
\hline & 30 & CM & 28,10 & 9,80 & 3,00 & 321 & 1,5 \\
\hline \multirow{3}{*}{ 19/fev/04 } & 120 & $\mathrm{CM}$ & 30,10 & 7,89 & 26,85 & 401 & 5,8 \\
\hline & 120 & $\overline{C M}$ & 22,60 & 5,68 & 22,01 & 272 & 5,8 \\
\hline & 120 & GV & 26,50 & 1,93 & 25,88 & 272 & 5,8 \\
\hline \multirow{2}{*}{ 22/fev/04 } & 1320 & GV & 1,57 & 4,59 & 11,09 & 234 & 54,5 \\
\hline & 1320 & $\mathrm{CM}$ & 6,54 & 9,93 & 7,09 & 234 & 54,5 \\
\hline \multirow{3}{*}{ 20/jan/05 } & 60 & IAG-USP & 4,65 & 27,90 & 7,89 & 40 & 25,9 \\
\hline & 60 & CM & 12,00 & 87,90 & 3,73 & 40 & 25,9 \\
\hline & 60 & GV & 4,55 & 27,90 & 12,78 & 40 & 25,9 \\
\hline \multirow{2}{*}{$\begin{array}{l}04-05 / \\
a b r / 05\end{array}$} & 120 & GV & 5,80 & 5,02 & 22,19 & 110 & 49,6 \\
\hline & 120 & CM & 8,32 & 36,85 & 8,05 & 110 & 49,6 \\
\hline \multirow{2}{*}{ 21/abr/05 } & 120 & GV & 14,83 & 2,29 & 1,67 & 190 & 6,4 \\
\hline & 120 & $\overline{C M}$ & 11,40 & 21,82 & 2,66 & 190 & 6,4 \\
\hline \multirow{4}{*}{$\begin{array}{l}21-22 / \\
\text { maio/05 }\end{array}$} & \multirow{4}{*}{540} & GV & 1,96 & \multirow{4}{*}{2,90} & \multirow{4}{*}{9,66} & \multirow{4}{*}{316} & \multirow{4}{*}{22,5} \\
\hline & & GV & 1,84 & & & & \\
\hline & & GV & 2,02 & & & & \\
\hline & & GV & 2,03 & & & & \\
\hline \multirow{2}{*}{ 20/jun/05 } & 300 & GV & 7,53 & 1,58 & 12,30 & 40 & 12,3 \\
\hline & 300 & $\mathrm{CM}$ & 6,30 & 18,20 & 14,86 & 40 & 12,3 \\
\hline \multirow[b]{2}{*}{ 10/mar/06 } & 30 & GV & 10,73 & 7,79 & 6,68 & 155 & 1,5 \\
\hline & 30 & IAG-USP & 14,00 & 27,70 & 14,22 & 155 & 1,5 \\
\hline
\end{tabular}

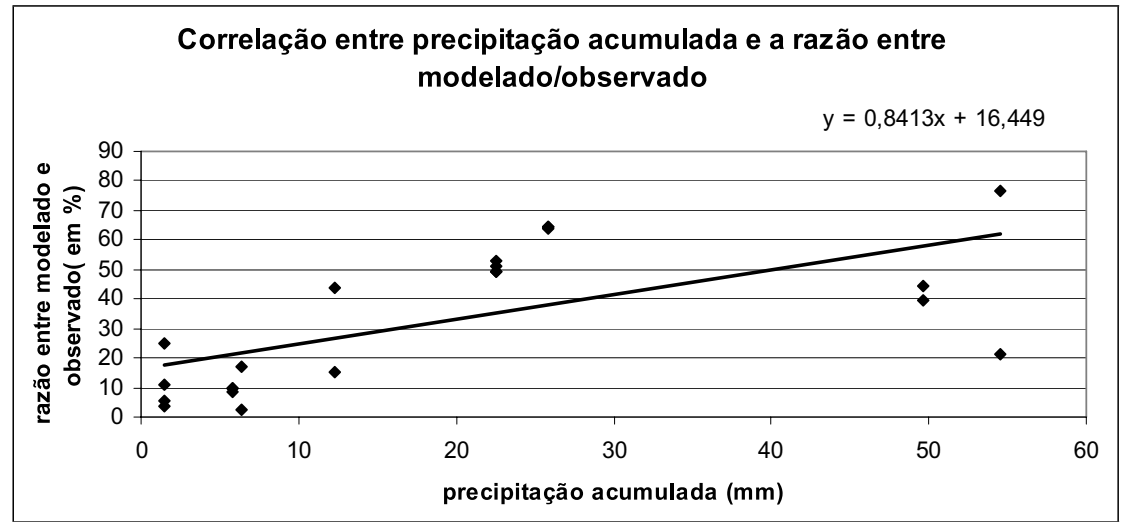

Figura 2 - Correlação entre a precipitação acumulada e a razão entre as concentrações de sulfato modeladas e observadas em água de chuvas por evento. Coeficiente de correlação de 0,63.

apenas quatro eventos (dos 19 estudados), os resultados de simulação representam pelo menos $40 \%$ da medida observada, os quais correspondem aos eventos do dia 22 de fevereiro de 2004 (Granja Viana), 20 de janeiro de 2005 (Granja Viana, Consolação/Mackenzie e IAG-USP), 4 e 5 de abril de 2005 (GV e
CM) e 21 e 22 de maio de 2005 (GV), todos convectivos. Única exceção seria o evento ocorrido na CM no dia 20/jun/05, com $44 \%$. Neste dia, houve precipitação total de 12,3 mm na Água Funda, correspondente ao maior volume entre os eventos mais fracos e mais estratiformes. 
Tabela 3 - Resultados da modelagem para cada evento de processos abaixo da nuvem (B) e intra-nuvem (C), comparando ao sulfato observado em água de chuva (A). A saída do modelo é a concentração de $\mathrm{SO}_{4}^{2-}$ na água em $\mu$ mol.L ${ }^{-1}$. A coluna \% ilustra a razão entre a modelada abaixo da nuvem (B) pela observada (A) e a porcentagem da razão entre a modelada intra-nuvem (C) e a observada (A). GV é Granja Viana e CM é Consolação/Mackenzie. Observar que os dados apresentados para o sulfato na água de chuva estão em $\mu \mathrm{mol} . \mathrm{L}^{-1}$.

\begin{tabular}{|c|c|c|c|c|c|c|}
\hline Eventos & $\begin{array}{l}\text { Local } \\
\text { de coleta }\end{array}$ & 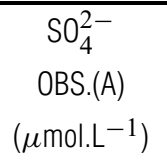 & $\begin{array}{c}\mathrm{SO}_{4}^{2-} \\
\mathrm{MOD} .(\mathrm{B}) \\
\left(\mu \mathrm{mol} . \mathrm{L}^{-1}\right)\end{array}$ & $\begin{array}{c}B / A \\
\%\end{array}$ & $\begin{array}{c}\mathrm{SO}_{4}^{2-} \\
\mathrm{MOD} .(\mathrm{C}) \\
\left(\mu \mathrm{mol} . \mathrm{L}^{-1}\right)\end{array}$ & $\begin{array}{l}\mathrm{C} / \mathrm{A} \\
\%\end{array}$ \\
\hline \multirow{2}{*}{ 12/fev/04 } & GV & 28,40 & 0,29 & 1,00 & 1,5 & 5,4 \\
\hline & CM & 28,10 & 0,27 & 0,95 & 1,1 & 3,8 \\
\hline \multirow{3}{*}{ 19/fev/04 } & CM & 30,10 & 0,72 & 2,40 & 2,9 & 9,6 \\
\hline & $\mathrm{CM}$ & 22,60 & 0,58 & 2,60 & 2,3 & 10,0 \\
\hline & GV & 26,50 & 0,58 & 2,80 & 2,3 & 8,7 \\
\hline \multirow{2}{*}{ 22/fev/04 } & GV & 1,57 & 0,04 & 2,50 & 1,2 & 76,4 \\
\hline & CM & 6,54 & 0,35 & 5,40 & 1,4 & 21,4 \\
\hline \multirow{3}{*}{ 20/jan/05 } & IAG-USP & 4,65 & 0,64 & 13,00 & 3,0 & 64,0 \\
\hline & $\begin{array}{c}\text { Consolação/ } \\
\text { Mackenzie }\end{array}$ & 12,00 & 1,60 & 13,00 & 7,6 & 64,5 \\
\hline & GV & 4,55 & 0,74 & 16,00 & 3,4 & 74,7 \\
\hline \multirow{2}{*}{$\begin{array}{l}04-05 / \\
\text { abr/05 }\end{array}$} & GV & 5,80 & 0,56 & 9,70 & 2,3 & 39,3 \\
\hline & CM & 8,32 & 0,93 & 11,00 & 3,7 & 44,5 \\
\hline \multirow{2}{*}{ 21/abr/05 } & GV & 14,83 & 0,08 & 0,56 & 0,33 & 2,2 \\
\hline & CM & 11,40 & 0,51 & 4,50 & 2,0 & 17,0 \\
\hline \multirow{4}{*}{$\begin{array}{l}\text { 21-22/ } \\
\text { maio/05 }\end{array}$} & GV & 1,96 & \multirow{4}{*}{0,26} & \multirow{4}{*}{13,00} & \multirow{4}{*}{1,0} & 51,0 \\
\hline & GV & 1,84 & & & & 53,0 \\
\hline & GV & 2,02 & & & & 49,5 \\
\hline & GV & 2,03 & & & & 49,3 \\
\hline \multirow{2}{*}{ 20/jun/05 } & GV & 7,53 & 0,29 & 3,80 & 1,2 & 15,0 \\
\hline & CM & 6,30 & 0,66 & 11,00 & 2,8 & 44,4 \\
\hline \multirow{2}{*}{ 10/mar/06 } & GV & 10,73 & 0,30 & 2,80 & 1,2 & 11,1 \\
\hline & IAG-USP & 14,00 & 0,86 & 6,10 & 3,5 & 25,0 \\
\hline
\end{tabular}

Adicionalmente, se forem tirados os eventos com pouca precipitação acumulada no IAG (valores menores que $20 \mathrm{~mm}$ ), ou seja, os eventos dos dias 12/fev/04, 19/fev/04, 21/abr/05, 20/jun/05 e 10/mar/06, todos com menos de 13 mm, o coeficiente de correlação entre modelado e observado passa a ser de 92\% $(\mathrm{P}<0,05)$ (ver Fig. 3), evidenciando assim, que os eventos claramente convectivos são bem melhor modelados.

Quanto às situações sinóticas dominantes, baseadas no METAR, primeiramente, pode-se notar que há uma dominância de entradas de frente frias, associadas à situação pós-frontal com os supracitados eventos convectivos e estratiformes. Não houve nenhum caso de convectivo isolado. Na média, os eventos de verão estudados forneceram resultados semelhantes aos de outono/inverno, ao redor de 30\%, com relação ao modelado e ao observado. Os eventos com grande diferença entre modelado e observado apresentam-se associados à situação pós-frontal, estratiforme e de fraca pluviosidade, todos com precipitação total de menos de $13 \mathrm{~mm}$. A correlação entre modelado e observado, nestes eventos, não é significativa nem claramente positiva, $(r=0,08)$. 


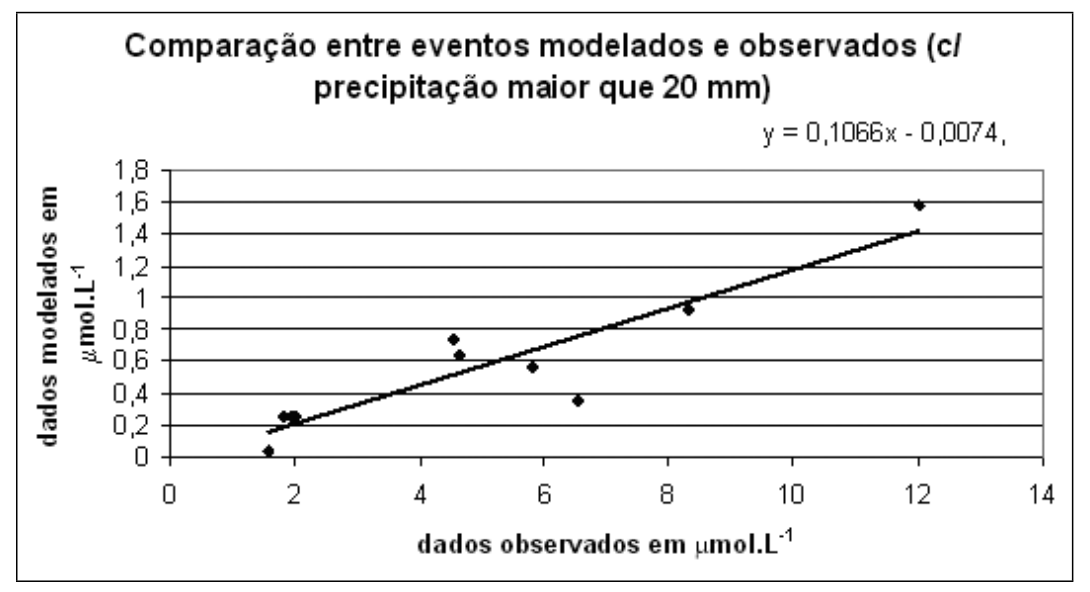

Figura 3 - Comparação entre eventos modelados e observados para precipitações acumuladas (no IAG-Água Funda) maiores que $20 \mathrm{~mm}$ com coeficiente de correlação de 0,92.

Portanto, no geral, nota-se que nestes eventos mais estratiformes com menor precipitação total, os valores da modelagem estão subestimados e que os desvios em relação à média são da mesma ordem de grandeza que o valor medido. Além deste fato, há diversas aproximações que induzem a erros no processo de modelagem tais como: não considerar advecção de massa entre as localidades e ao fato de os dados de entrada não estarem exatamente no local e no tempo requeridos (Gonçalves et al., 2007a). Outra explicação poderia ser devido ao tamanho real da nuvem (utilizado valor fixo de $8000 \mathrm{~m}$ ). 0 oposto pode-se dizer dos eventos claramente convectivos, onde o provável grande tamanho da nuvem elevou o coeficiente de correlação entre modelado e observado nestes eventos.

\section{Comparação entre as localidades}

Utilizando agora apenas os dados referentes às diferenças entre as localidades, foi construída a Tabela 4. Nesta tabela, os valores médios modelados estão abaixo dos observados em ambas as localidades. Adicionalmente, as correlações entre ambas, com todos os eventos observados, são estatisticamente significativas, ao redor de 0,94 $(P<0,05)$. Novamente, os eventos mais convectivos elevam o coeficiente de correlação em uma comparação entre as localidades. No entanto, a despeito desta relação, entre os modelados para ambas as localidades, 0 coeficiente de correlação cai para 0,42, 0 que denota, novamente, a inclusão de eventos de fraca pluviosidade, com menor qualidade de simulação. Nesta tabela, as médias da concentração observada entre as duas localidades estão próximas (diferença de 5,0 $\mu$ mol... ${ }^{-1}$ ) bem como as médias da concentração modelada (diferença de $1,7 \mu \mathrm{mol} . \mathrm{L}^{-1}$ ). Ainda que os valores modela- dos estejam aquém do observado, pelas razões acima expostas, 0 valor modelado total do CM é superior ao da GV por um fator de 1,85, enquanto que na concentração observada esta diferença se reduz para 1,45, enfatizando as diferenças apresentadas pelos coeficientes de correlação para os eventos observados e para os modelados. Ambas as concentrações, modeladas e observadas, em geral, são maiores na região central da RMSP do que na periférica, o que era de se esperar, pelas concentrações dos dados de entrada. Isto significa que pode não haver muita advecção de massa dentro da região de RMSP, notabilizado por estas diferenças.

Tabela 4 - Médias das concentrações de sulfato observadas e modeladas total e por localidade.

\begin{tabular}{|l|c|c|c|}
\hline \multirow{2}{*}{} & \multicolumn{3}{|c|}{ Média $\left(\mu \mathrm{mol} . \mathrm{L}^{-1}\right)$} \\
\cline { 2 - 4 } & $\begin{array}{c}\text { Todos os } \\
\text { eventos }\end{array}$ & GV & CM \\
\hline Observações & 13,00 & 11,0 & 16,0 \\
\hline Modelado total & 2,90 & 2,0 & 3,7 \\
\hline Modelado Abaixo da nuvem & 0,54 & 0,4 & 0,7 \\
\hline Modelado Intra-nuvem & 2,40 & 1,6 & 3,0 \\
\hline
\end{tabular}

Nestes eventos, contudo, deve-se salientar que há eventos em que estas diferenças não se estabeleceram, notadamente, nos eventos dos dias 12/fev/04, 21/abr/05 e 20/jun/05. Nestes eventos a concentração observada na GV foi mais alta que a no CM, onde no primeiro evento (12/fev/04) a concentração modelada foi igualmente superior à GV. Em geral, estes eventos estão associados a sistemas com pouca precipitação, nos eventos do dia 12/fev/04, onde choveu $30 \mathrm{ml}(1,5 \mathrm{~mm}$ de precipitação acumulada, Tab. 2) e do dia 20/jun/05, onde choveu $40 \mathrm{ml}$ (12,3 mm 
de precipitação acumulada), são os menores valores obtidos de uma média de $168 \mathrm{ml}$, 0 que indica sua origem em nuvens estratiformes, de baixa altitude. Os dados de precipitação do IAG suportam este resultado, com precipitações totais menores que $13 \mathrm{~mm}$ como citado acima. Este resultado necessita de uma meIhor investigação com um modelo de mesoscala, como o BRAMS, onde a altura da nuvem e os processos advectivos são mais eficientemente simulados, já que as mesmas diferenças são encontradas nos dados observados. 0 evento do dia 21/abr/05 foi, aparentemente, uma exceção, onde o volume coletado foi de $190 \mathrm{ml}$, entretanto, no IAG a precipitação total não ultrapassou $6,4 \mathrm{~mm}, 0$ que reforça 0 argumento que foi precipitação leve no geral.

Conseqüentemente, para ambas as localidades, o efeito predominante é 0 intra-nuvem (cerca de $80 \%$ para todos eventos e localidades), como era esperado para uma região subtropical como a RMSP. Os resultados relativos à comparação entre as localidades sugerem a dominância do processo intra-nuvem em ambas, sendo o processo de remoção gerado por somente um sistema estratiforme. Portanto, os resultados aqui apresentados são corroborados pela Gonçalves et al. $(2002,2003)$ e por Nogushi et al. (2007).

\section{CONCLUSÕES}

Em geral, o modelo subestima as concentrações de $\mathrm{SO}_{4}^{2-}$ dos processos abaixo da nuvem. Contudo destaca-se que a remoção de poluição intra-nuvem é mais eficiente do que 0 abaixo da nuvem sendo as concentrações modeladas da ordem de 4,6 vezes maiores, ainda assim aquém das concentrações observadas. Estas diferenças podem ser devido a diversos fatores tais como: não advecção de massa e a altura da nuvem fixada em 8000 m, requerendo um estudo mais aprofundado com uso de modelos de mesoscala.

Eventos claramente convectivos, com precipitação total maior que $20 \mathrm{~mm}$, são mais eficazmente modelados que os eventos mais fracos, com um coeficiente de correlação de 0,92. Há também uma clara associação entre eventos de maior precipitação e a razão entre modelado e observado.

Como conclusão geral, pode-se afirmar que para este conjunto de eventos, de diferentes estações do ano, nota-se uma predominância da remoção do tipo intra-nuvem em cerca de 80\% contra cerca 20\% de remoção abaixo da nuvem. Estes resultados são diferentes dos obtidos nos dois eventos no trabalho anterior com respeito à RMSP (Gonçalves et al., 2007a). Nos dois eventos neste artigo, houve domínio dos processos abaixo da nuvem. Portanto, este presente artigo ilustra um número maior de eventos, enfatizando o domínio dos processos intra-nuvem, em desacordo com os resultados deste artigo supracitado. Os resultados requerem um refinamento da modelagem bem como uma maior coleta de dados.

Os valores das concentrações médias na região periférica da RMSP, na Granja Viana, apresentaram concentrações menores tanto no ar, (conseqüentemente na água de chuva modelada) como na água de chuva observada, como seria de se esperar devido à distância das fontes de poluição. No entanto, houve eventos que esta relação não foi obedecida, a região periférica apresenta valores superiores ao da região central. Isto significa que pode não ter tido muita advecção de massa dentro da região de RMSP, notabilizado por estas diferenças. Este resultado pode ser devido a eventos de baixa precipitação (estratiformes) com uma distribuição mais eqüitativa de poluentes na chuva em áreas significativamente maiores. Contudo, as diferenças e semelhanças entre as localidades sugerem a dominância do processo intranuvem em ambas as localidades. Deve-se salientar que estes eventos correspondem a cerca de $40 \%$ do total de eventos na RMSP e $61 \%$ do total de chuva acumulado de acordo com a literatura (Morales \& da Rocha, 2008).

Como perspectivas, faz-se necessário esclarecer as diferenças entre estes eventos e as localidades. Este esclarecimento pode ser feito através de modelagem numérica usando 0 modelo RAMS ou BRAMS (Brazilian Regional Atmospheric Modeling System), para calcular a advecção de massa bem como um maior detalhamento dos processos intra-nuvem, como a altura da base e topo da mesma.

\section{AGRADECIMENTOS}

À FAPESP (no. 06/50099-0) pela bolsa de Iniciação Científica ao aluno Luiz Carlos Mantovani Jr. e à MSc. Rosana Astolfo, técnica do LAPAt.

\section{REFERÊNCIAS}

ANDRONACHE C. 2002. Estimated variability of below-cloud aerosol removal by rainfall for observed aerosol size distributions. Atmospheric Chemistry and Physics Discussions, 2: 2095-2131.

ASMAN WAH. 1995. Parameterization of below-cloud scavenging of highly soluble gases under convective conditions. Atmos. Environ., 29: $1359-1368$.

BRAGA ALF, CONCEIÇÃO GMS, PEREIRA LAA, KISHI HS, PEREIRA JCR, ANDRADE MF, GONÇALVES FLT, SALDIVA PHN \& LATORRE MRDO. 2000. Air pollution and pediatric respiratory hospital admissions in São Paulo, Brazil. J. Environm. Medicine, 1: 95-102.

CALDERÓN SM, POOR ND \& CAMPBELL SW. 2007. Estimation of the particle and gas scavenging contributions to wet deposition of organic nitrogen. Atmos. Environ., 41: 4281-4290. 
CHAMBERLAIN AC. 1953. Aspects of travel and deposition of aerosol and vapor clouds. British report AERE-HP/R-1261.

CHRISTOPHER MBL, C VAN BOWERSOX, LARSON R \& LARSON SM. 2007. Monitoring log-term trends in sulfate and ammonium in US Precipitation: results from the National Atmospheric Deposition Program/National Trends Network. Water, Air, Soil Pollut., 7: 59-66.

FORNARO A, ROCHA FR, FRACASSSI DA SILVA JA, LAGO CL \& GUTZ IGR. 2003. Acid Acid deposition and related atmospheric chemistry in the São Paulo Metropolis, Brazil: Part I. Capillary electrophoresis of rainwater and long term trends of $\mathrm{pH}$ and ionic composition. Atm. Environ, 37: 105-115.

FORNARO A \& GUTZ IGR. 2006. Wet deposition and related atmospheric chemistry in the São Paulo metropolis, Brazil: Part 3. Trends in precipitation chemistry during 1983-2003 period. Atmos. Environ., 40: 5893-5901.

FORTI MC, MOREIRA-NORDEMANN LM, ANDRADE MF \& ORSINI CQ. 1990. Elements in the precipitation of S. Paulo City (Brazil). Atm. Environ., 24B: 355-360.

GONÇALVES FLT, MALHEIROS AR, FREITAS RS, ASSUNÇÃO MAF \& MASSAMBANI 0. 2002. In-cloud and below-cloud numerical simulation of scavenging processes at Serra do Mar region, SE Brazil. Atm. Environ. 36(33): 5245-5255.

GONÇALVES FLT, ANDRADE MF, FORTI MC, ASTOLFO R, RAMOS AM, MASSAMBANI 0 \& MELFI AJ. 2003. Preliminary estimation of the rainfall chemical composition evaluated through the scavenging Modeling for North-Eastern Amazonian region (Amapá State, Brazil). Environmental Pollution, 121(1): 63-73.

GONÇALVES FLT, NAKAEMA WN, ANDRADE MF \& FORNARO A. 2007a. In-cloud and below-cloud scavenging analysis of sulfate in the metropolitan area of São Paulo, Brazil. Revista Brasileira de Meteorologia, 22(1): 94-104.

GONÇALVES FLT, BRAUN S, SILVA DIAS PL \& SHAROVSKY R. 2007b. Influences of the weather and air pollutants on cardiovascular disease in the metropolitan area of São Paulo. Environmental Research, 104: 275281.

HERBERT F \& BEHENG KD. 1986. Scavenging of airborne particles by collision with water drops - Model studies on the combined effect of essential microdynamic mechanisms. Meteorol. Atm. Phys., 35: 201-211.

HILL FB \& ADAMOWICZ RF. 1977. A model for rain composition and the weather of sulphur dioxide. Atmos. Environ., 11: 917-927.

LEAL TFM, FONTENELE APG, PEDROTTI JJ \& FORNARO A. 2004. Composição iônica majoritária de águas de chuva na região central de São Paulo. Química Nova, 27(6): 855-861.

MIRCEA M, STEFAN S, FACCHINI MC \& FUZZI S. 2004. Analytical formulas for the below-cloud scavenging coefficient of an irreversibly soluble gas: a quantitative evaluation for $\mathrm{HNO}_{3}$. International Journal of Environment and Pollution, 21: 547-565.

MORALES CA \& DA ROCHA RP. 2008. Does the pollution affect the development of the thunderstorms over the city of São Paulo, Brazil? $15^{\text {th }}$ Internation. Conference on Cloud and Precipitation, July 7-11, 9-10.

MOREIRA-NORDEMANN LM, PALOMBO CR, BERTOLI JLR \& CUNHA RC DE A. 1983. Análise química preliminar das águas de chuva de Cubatão - Impactos ambientais. Instituto Nacional de Pesquisas Espaciais, $12 \mathrm{pp}$.

NAKAEMA WM. 2002. Modelagem da deposição úmida de poluentes atmosféricos na grande São Paulo durante os meses de inverno. Dissertação de Mestrado defendida no DCA-IAG-USP, 210 pp.

NOGUSHI I, KENTARO H, MASAHIDE A, OHIZUMI T, MINAMI Y, KITAMURA M, TAKAHASHI A, TANIMOTO H \& HARA K. 2007. Temporal Trends of Non-sea Salt Sulfate and Nitrate in Wet Deposition in Japan. Water, Air and Soil Pollut., 7: 67-75.

OKITA T, HIROSHI H \& FUZUKAZI N. 1996. Measurements of atmospheric $\mathrm{SO}_{2}$ and $\mathrm{SO}_{4}^{2-}$, and determination of the wet scavenging coefficient of sulphate aerosols for the winter monsoon season over the sea of Japan. Atm. Environ., 30(22): 3733-3739.

PRUPPACHER HR \& KLETT JD. 1997. Microphysics of clouds and precipitation. Ed. Reidel Publ. Company, 714 pp.

SALDIVA PHN, KING M, DELMONTE VLC, MACCHIONE M, PARADA MAC, DALIBERTO ML, SAKAE RS, CRIADO PMP, PARADA PLP, ZIN WA \& BÖHM GM. 1992. Respiratory alterations due to urban air pollution an experimental study in rats. Environ. Res., 57: 19-33.

SANTOS MA, ILLANES CAF, FORNARO A \& PEDROTTI JJ. 2007. Acid Rain in the central region of São Paulo City - Brazil. Water, Air \& Soil Pollution: Focus, 7(1): 85-92.

SEINFELD JH \& PANDIS SN. 1998. Atmospheric and physics of air polIution. Ed. John Wiley \& Sons, 1030 pp.

SHAROVSKY R. 2001. Efeitos da temperatura e poluição do ar na mortalidade por infarto agudo do miocárdio no município de São Paulo. Tese (Doutorado) - Faculdade de Medicina, Universidade de São Paulo, São Paulo. 86 pp.

SILVA MPR, GONÇALVES FLT \& FREITAS S. 2009. Two case studies of sulfate scavenging processes in the Amazon region (Rondônia). Environmental Pollution, 157: 637-645.

TERAUDA E \& NIKODEMUS 0. 2007. Sulphate and nitrate in precipitation and soil water in Pine Forests in Latvia. Water, Air and Soil Pollut., 7: $77-84$

YNOUE RY \& ANDRADE MF. 2004. Size resolved mass balance of aerosol particles over the Metropolitan Region of São Paulo, Brazil. Journal of Aerosol Science and Technology, 38: 52-62. 


\section{NOTAS SOBRE OS AUTORES}

Fábio Luiz Teixeira Gonçalves. Possui mestrado em Ciências Biológicas (Botânica) pela Universidade de São Paulo (1990) doutorado e livre-docência (2006) em Meteorologia pela Universidade de São Paulo (1997). Atualmente é professor MS-5 da Universidade de São Paulo. Tem experiência na área de Geociências, com ênfase em Meteorologia Ambiental, atuando principalmente nos seguintes temas: modelagem numérica, análise de componentes principais, epidemiologia, poluição atmosférica e biometeorologia.

Luiz Carlos Mantovani Junior. Bacharel em Meteorologia pela Universidade de São Paulo, formado em 2008. Bolsista Fapesp entre 2006 e 2007 para desenvolvimento de trabalho sobre modelagem de remoção de poluentes atmosféricos na cidade de São Paulo. Trabalha com Risco de Mercado Financeiro na empresa MAPS Soluções e Serviços como Analista Financeiro na equipe responsável pela implantação do sistema e consultoria. Desenvolve relatórios de risco customizados para tesourarias e assets bem como acompanha estruturação da área de risco dos clientes. Atualmente cursa MBA - Master in Economics na Fundação Getúlio Vargas.

Adalgiza Fornaro. Possui mestrado em Química Analítica pela Universidade de São Paulo, IQ-USP (1991) e doutorado em Química Analítica pela Universidade de São Paulo, IQ-USP (1996). Atualmente é docente no Departamento de Ciências Atmostéricas do Instituto de Astronomia, Geofísica e Ciências Atmosféricas (IAG-USP). Tem experiência na área de Química, com ênfase em Química da Atmosfera, atuando principalmente nos seguintes temas: poluição do ar, chuva ácida, composição química de águas de chuva e material particulado, oxidantes atmosféricos (ozônio e peróxido de hidrogênio), ácidos carboxílicos, processos de remoção de poluentes atmostéricos.

Jairo José Pedrotti. Possui graduação em Química Industrial pela Universidade Federal de Santa Maria (1983), mestrado em Química Analítica pela Universidade de São Paulo (1987) e doutorado em Química Analítica, IQ-USP (1993). Tem estágio de pós-doutoramento na Divisão de Química Analítica do CENA-USP entre 1994 e 1996. Atualmente é professor titular da Universidade Presbiteriana Mackenzie e responsável pelo Laboratório de Química Analítica Instrumental. Tem experiência na área de Química, com ênfase em Eletroanalítica e Química Analítica Ambiental, atuando principalmente nos seguintes temas: análise por injeção em fluxo, detectores eletroquímicos, instrumentação analítica, avaliação de poluentes atmosféricos em águas de chuva e material particulado 